\title{
Asian upper blepharoplasty
}

\author{
Juwan Park, MD, PhD', Changhyun Koh, MD¹, Woongchul Choi, MD, PhD² \\ ${ }^{1}$ Department of Ophthalmology, Yeouido St. Mary's Hospital, The Catholic University of Korea, Rep. of Korea, ${ }^{2}$ Myoung Eye Plastic Surgery \\ Center, Seoul, Rep. of Korea
}

\begin{abstract}
Asian eyelids have different anatomical structures compared to Western eyelids; accordingly, the standards for the "beauty of eyes" vary among races and countries and have also changed with time. With increasing interest in cosmetic surgery, Korean cosmetic techniques and beauty standards have become wide spread in China and other Southeast Asian countries due to the influence of the Korean wave. The recent trend of beauty is naturalism. Although cosmetic operations are being performed, the inconvenience caused by the operation should be minimal, and a natural look is preferred. That is, minimally invasive surgery is necessary. To minimize unnecessary complications and to improve cosmetic and functional satisfaction with minimal invasion and uncomplicated methods according to the individual patient's condition, we must thoroughly understand the characteristics of eyelid anatomy and function of Asian eyelids. Therefore, we describe the surgical procedure for successful upper eyelid surgery based on the characteristics of Asian eyelids.

Level of Evidence: $\mathrm{V}$
\end{abstract}

Keywords: Asian eyelids; cosmetic surgery; a natural look; minimally invasive surgery

\section{Introduction}

\section{Change of concept}

Blepharoplasty is the most commonly performed plastic surgery and non-incision double eyelid surgery was introduced for the first time in the late 19th century in Japan, as an improvement on the conventional incision technique. Various methods of non-incision surgery have since been introduced [1]. The first attempt was to make a three-point suture with $3 \mathrm{~mm}$ intervals and 6 to $8 \mathrm{~mm}$ heights from the lid margin and to loosen it 4 to 6 days after surgery. As a result, the double eyelids were small and appeared at the level of the tarsus.

Thereafter, the incision technique was introduced by Khoo Boo-Chai in Singapore. The height of the double eyelid was 10 to $12 \mathrm{~mm}$, which was not suitable for Asian people. In Korea, double eyelid surgery was performed by a US military doctor after the Korean War, and a very large lid crease was popular at that time. However, a high supratarsal fixation with lid crease at a very high position caused an unnatural look, as if the eyes of a Westerner were placed on the face of an Asian, with a sleepy appearance (Fig. 1).

After the 1990s, it has become more favorable to offer natural surgical results. With the popularity of the Korean Wave, Korean cosmetic surgical techniques and beauty standards have influenced Asia and other countries. However, despite the recent tendency to pursue naturalistic beauty perspectives, some Korean plastic surgeons pursue more dramatic changes. They advertise that they can enlarge eyelids in four directionsup, down, medial and lateral-to make eyes bigger and more beautiful. However, these unusual and unreasonable surgeries 


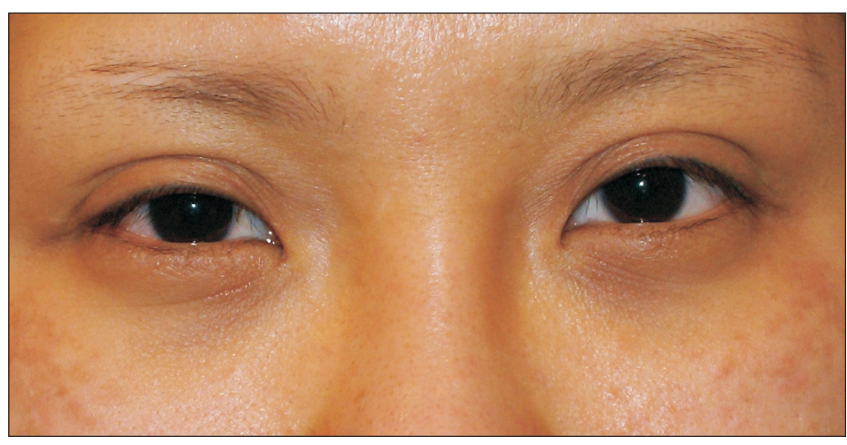

Fig. 1. 31 This 31-year-old woman has had previous upper blepharoplasty, but she looks droopy because of large lid crease.

ultimately increase the possibility of complications. Therefore, surgeons should consider the method and type of operation with the aim of avoiding complications as much as possible, considering the individual condition of the patient. For example, in the case of lateral canthoplasty, the effect is minimal and the surgery can cause eye discomfort due to aggravation of dry eye. In addition, many complications such as granuloma, redness, scarring, adhesion, canthal webbing, trichiasis, distichiasis, ectopic lacrimal fistula, symblepharon, entropion, and ectropion are frequently encountered, requiring reoperation. Therefore, the surgeon should carefully choose the surgical technique from a conservative point of view.

\section{Mechanism of lid crease formation}

It is widely known that there is a difference in lid crease formation due to anatomical differences between the eyelids of Asian and Western people [2].

For Western women, the height of the lid crease is about 9-11 $\mathrm{mm}$ from the lid margin and slightly lower $(7-8 \mathrm{~mm})$ on the medial side [3]. Asian people usually have no lid crease or a height much lower than that of Westerners. The reason for this is that the levator aponeurosis attaches very low to the orbital septum or there is no attachment to the eyelid skin [4].

The most common theory for the formation of the lid crease is the levator expansion theory [5]. According to some reports, the attachment of superficial fibers of the levator aponeurosis to the skin causes wrinkles and lid creases [6-8]. Likewise, it was reported that Asian people had more subcutaneous fat and retro-orbicularis oculi fat, and that pre-aponeurotic fat is located in front of the tarsal plate because the orbital septum meets the levator aponeurosis at the lower tarsal border [9]. However, through a cadaveric study it was reported that the formation of lid crease is caused by the thickness of skin and the orbicularis oculi muscle (OOM) in the area of the lid crease [10].

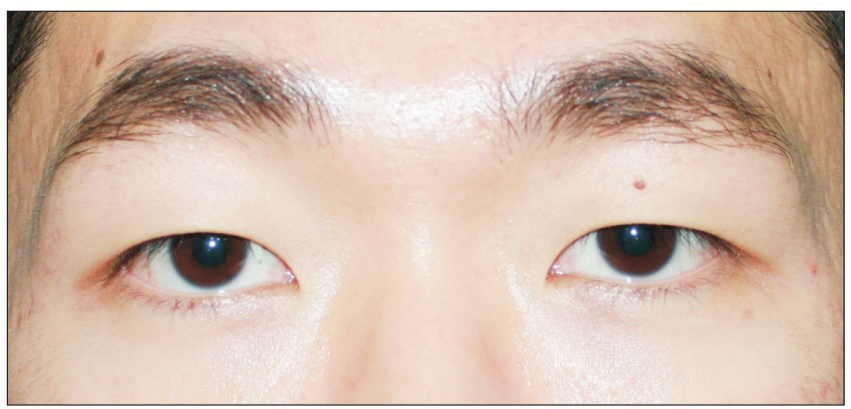

Fig. 2. This young man shows frontalis hyperaction due to superior visual field disturbance and the lash ptosis by the overhanging upper lid skin.

\section{Characteristics of Asian upper eyelids}

The eyelids of Asians without lid crease take various forms depending on anatomical characteristics. These are the degree of proptosis or enophthalmos, the degree of protrusion of the superior and inferior orbital rim, the thickness of the skin and OOM, the presence of orbital fat prolapse, the amount of subbrow fat, and the difference in the positions of the medial and lateral canthal tendon. Prior to surgery, the surgeon can determine the characteristics of each patient through preoperative examination and explain the predicted results to the patient in advance, thereby improving the patient's understanding and satisfaction.

Though there might be a thin eyelid without a double eyelid, the typical eyelid features of Asians are puffiness and frontalis hyperactivity (Fig. 2). The cause of this puffiness (fullness) is thick skin, OOM, fat, and sub-brow fat. If there is no lid crease, eyelashes can be depressed, and the visual field can be blocked by the overhanging skin. If the surgeon corrects this functional problem, the habit to raise the eyebrows using the forehead muscles is improved, and the inwardly depressed eyelashes can be turned outward, reducing discomfort. If the epicanthal fold, which is another characteristic of Asians, is severe, the inner eyelash sags inward, and the eyes become narrow. When this trait is corrected by medial epicanthoplasty, the direction of the inner eyelashes is corrected and the ratio of interpalpebral fissure to horizontal eye length of both eyes is improved, producing a good cosmetic result.

\section{Preoperative considerations}

\section{Preoperative evaluation}

Thorough preoperative evaluation is essential for good results. First, we assess the history of facial paralysis, eye trauma, surgery, and other procedures on the eye and determine 
whether the eyelids, eyebrows, and forehead wrinkles are asymmetric. In addition, we evaluate forehead hyperactivity, forehead wrinkles, and deeper wrinkles in upward gaze.

The marginal reflex distance-1 (MRD-1), the IPF (interpalpebral fissure), the LF (levator function), and the locations of the eyebrows and position of the lower eyelid should be recorded (Fig. 3). If the upper eyelid looks puffy, grasp the eyelid with the thumb and index finger to distinguish whether it is due to the thickness of skin and muscle or that of fat (Fig. 4). Fat slides between the fingers and moves backward, while skin and muscles are grasped and thus can be estimated. If the skin and muscles are not thick and the fat is large, the postoperative result will be relatively good. However, if the skin and muscles are thick, swelling can be relatively long-lasting, and surgical scars can be prominent. The infrabrow area can still appear puffy. Patients should be advised on the likely unfavorable effects after surgery, since some complain that their eyelids are puffy, even after hearing these explanations before surgery. In addition, skin

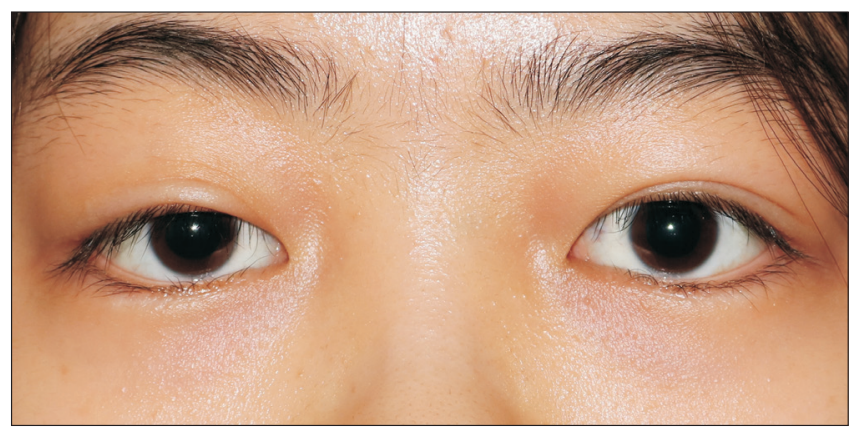

Fig. 3. This patient shows right droopy lid with brow elevation, and lower lid sag is noticeable on both eye.

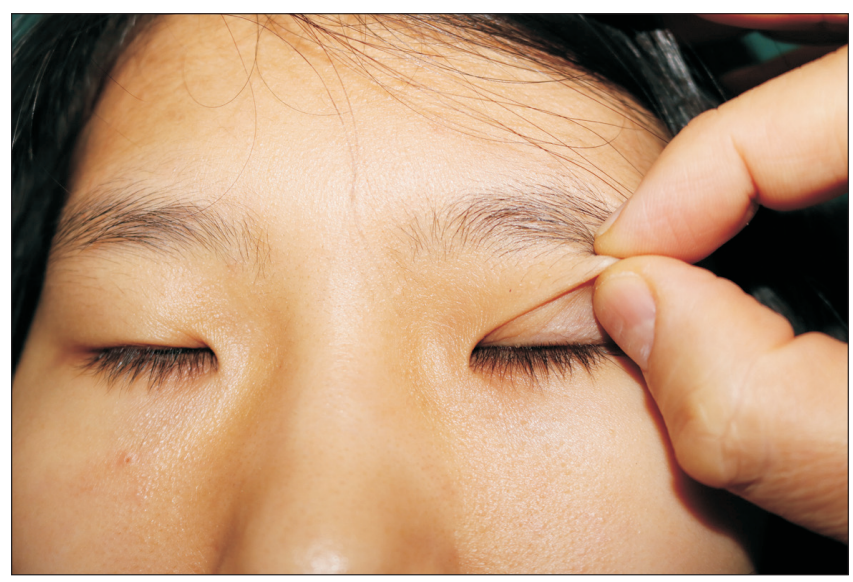

Fig. 4. The examiner grasps the upper lid with the thumb and index finger, and fat slides backward. In this patient, skin and muscle is not much thick. resection blepharoplasty in patients with brow ptosis can cause more prominent eyelid fullness, resulting in poor cosmetic results [11].

The beauty of each race is based on unique features that produce harmony, symmetry, and balance [12]. Therefore, the surgeon needs to know what factors impede harmonious, symmetrical, and balanced beauty during preoperative evaluation and explain the anticipated results to the patient in advance.

\section{Differential diagnosis}

One of the most common causes of postoperative asymmetry is the difference in eyelid level between eyes. When eyelid skin covered the upper lid margin, the difference between the two eyes might not be noticeable or can seem insignificant. If there is a difference in lower eyelid position despite the absence of previous history of facial paralysis, the difference between lower eyelid level in both eyes should be carefully examined.

An Asian single eyelid and dermatochalasis in an aged patient can cause pseudoptosis with a small eye due to redundant skin. A surgeon must distinguish between pseudoptosis, in which the eyes look small, and true ptosis, in which the eyelid margin truly fall. To do that, the concept of MRD-1, which is the distance from the light reflex of the cornea to the upper lid margin rather than the hanging upper lid fold, must be accurately understood and correctly measured. The examiner tells the patient to look straight without excessive use of the frontalis muscle and measures the distance from the corneal light reflex to the edge of the upper eyelid (Fig. 5). This makes it possible to distinguish between true ptosis and pseudoptosis. It is also possible to determine masked upper lid retraction associated with thyroid eye disease.

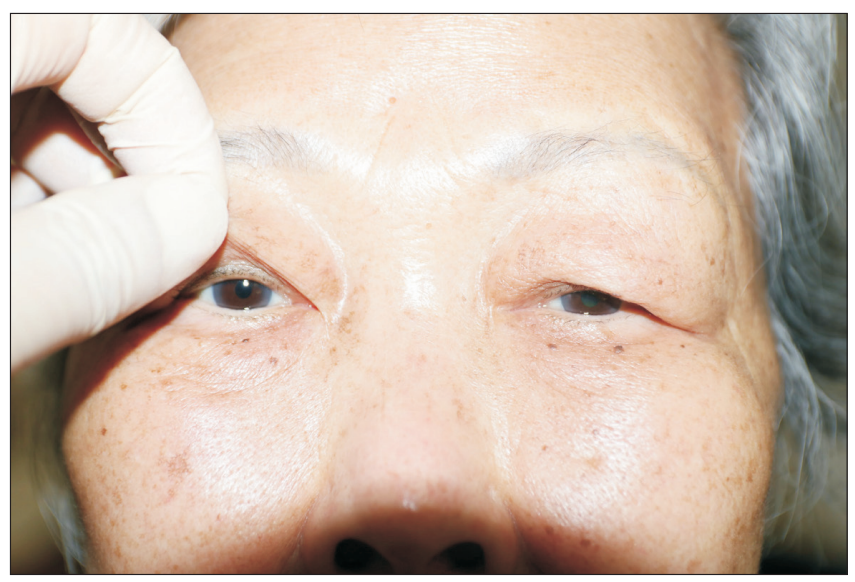

Fig. 5. By lifting the redundant upper lid skin, we can measure MRD1 accurately from the upper lid margin. 
If there is enophthalmos or exophthalmos, it can affect the outcome of the surgery, and if there is a difference in the degree of exophthalmos in each eye, it can cause asymmetry after surgery. Therefore, use the Hertel exophthalmometer to record the degree of exophthalmos in both eyes (Fig. 6) and to explain the effect to the patient before surgery. In the case of exophthalmos, protruding eyes after blepharoplasty can be seen more prominently. If there is enophthalmos due to microphthalmos or orbital wall fracture, the eyes look sleepy, and the interpalpebral fissure becomes smaller. Depending on the cause and severity, eyelid surgery can be performed after correcting for differences in the degree of exophthalmos through orbital surgery if necessary. If there is hypotropia, it is better to design the double eyelid line slightly lower than the opposite side because lid crease with hypotropia can seem large after surgery.

\section{Eyelash direction}

Lash ptosis is the overall lowering of the upper eyelid hair follicles located between Riolan's muscle and the pretarsal orbicularis muscles.

In a typical Asian eye with burden factor and no lid crease, there are no levator branches from the levator aponeurosis to the dermis, and eventually the pretarsal and preseptal OOM become loose. As a result, the skin and OOM droop and push down on the eyelashes. In addition, not only the length of the eyelashes seems shorter as the eyelashes are pressed and drooped (Fig. 7), but also the function of the eyelashes to protect the eyeball surface is impaired.

In a study of the degree of lash ptosis in Asian populations, Lee et al. found that when the degree of lash ptosis was graded as grade 0 ( 0 to 30 degrees over the horizontal plane), grade 1 ( 0 to 30 degrees under the horizontal plane), grade 2 (30 to 45 degrees under the horizontal plane), and grade 3 ( $>45$ degrees

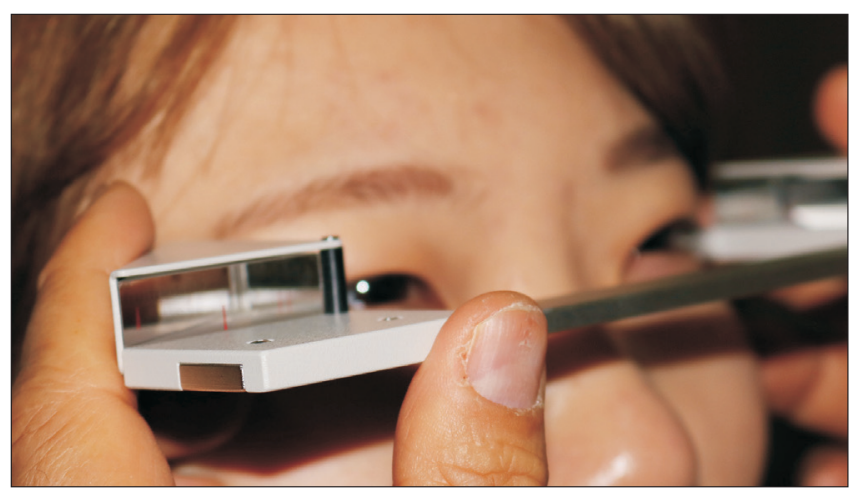

Fig. 6. The examiner is checking the extent of eyeball protrusion by Exophthalmometer. under the horizontal plane), the mean value was 1.88, meaning that the eyelashes of Asian people were close to 30 degrees below the horizontal plane. However, this classification has a large rating scale, therefore it can be difficult to distinguish among groups [13].

Before and after surgery, the direction of the eyelashes should be graded and recorded.

When the frontal view of the eyelid without the use of the forehead muscle was perpendicular to the vertical facial plane, it was defined as grade 1 . In comparison, grade 2 was 15 degrees below grade 1 , and grade 3 was 30 degrees below grade $1[13,14]$. Grade 1-2 is regarded as normal and is the aim of correction after surgery.

\section{Epicanthal fold}

The epicanthal fold is unique to Asian populations, resulting in a round-looking inner eye and a small horizontal palpebral fissure (Fig. 8) [15]. When performing blepharoplasty, medial epicanthoplasty can be used to expand the horizontal palpebral fissure to reduce the pinched appearance and make larger looking eyes to further improve cosmetic results [16]. On the other hand, in patients with a prominent epicanthal fold, blepharoplasty without correction for the epicanthal fold can result in a small, round, and surprised looking eye shape due to the short horizontal palpebral fissure and long intercanthal distance $[17,18]$.

The causes of epicanthal fold are various. Z-shaped twist of OOM fibers, excessive OOM and fibroadipose tissues, and absence or almost no medial attachment of levator aponeurosis

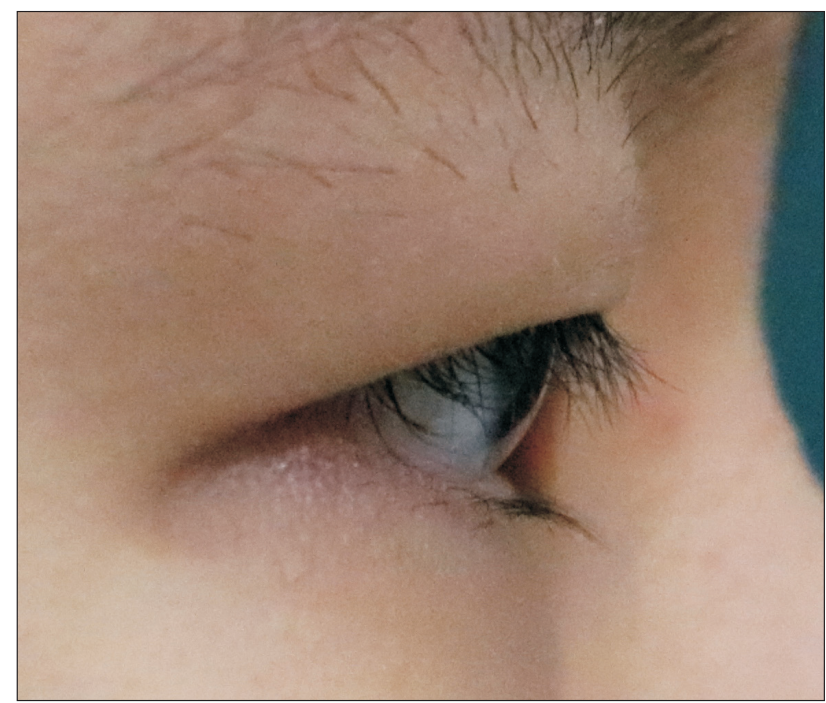

Fig. 7. This photo show grade 3-4 lash ptosis, upper eyelashes are pressed by the overhanging upper lid skin. 
have been thought to be causative factors of epicanthal fold $[19,20]$. Ten eyes of 7 cadavers with epicanthus tarsalis were analyzed and researchers found that epicanthal fold formation was dependent on intermuscular fibers of the preseptal orbicularis muscle in the oblique direction [21].

Reports show that as the medial attachment of the levator aponeurosis detaches, the epicanthus bulges in a convex shape and forms concave wrinkles. In addition, the preseptal OOM, which is thickened, is also displaced due to gravity, contributing to the formation of a convex shape [22].

The most common form of epicanthal fold is epicanthus tarsalis, which is clinically classified as type II using Park's classification, and it is the goal of surgery to make epicanthus palpebralis type II (Fig. 9). If the intercanthal distance is wide, medial

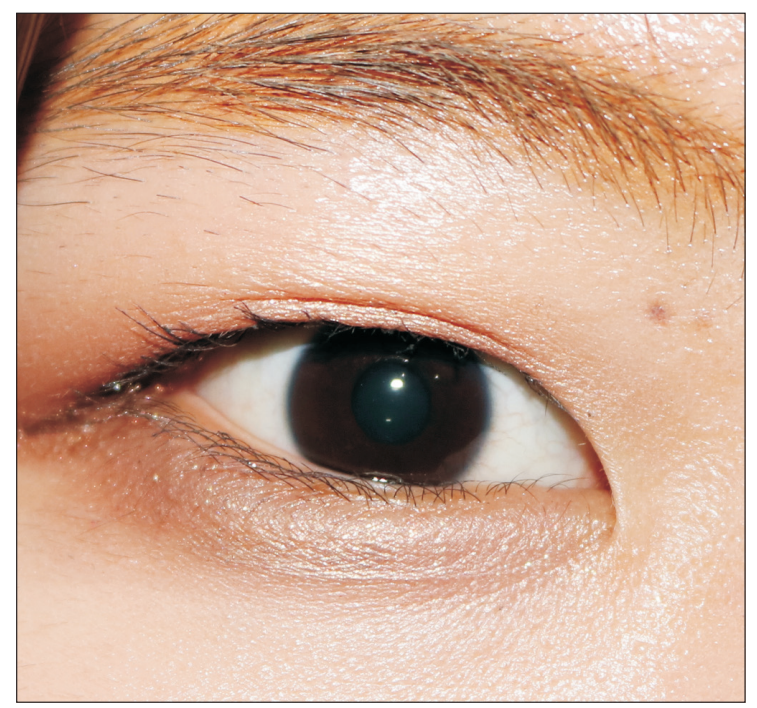

Fig. 8. This 21-year-old man shows round-looking inner eye and a small horizontal palpebral fissure due to the medial epicanthal fold. canthal tendon plication can be performed, and the intercanthal distance can be narrowed. However, excessive surgery can reduce patient satisfaction due to overexposure of the caruncle.

Various surgical methods have been introduced, usually based on Z-plasty and Y-V plasty. The author prefers Park Zepicanthoplasty because the design is easy, simple, and not time-consuming.

When the tension around the medial canthus is released after the skin incision, the skin muscle flap naturally rotates in Park Z-epicanthoplasty (Fig. 10). To confirm this and to minimize scarring, care should be taken to avoid tension during skin suture.

Recently, the skin redraping technique has been introduced and widely used. The advantage of this method is that it can be applied to any type of epicanthal fold without scarring because the incision is along the lid margin. However, care should be taken to avoid punctal eversion due to scar tissue or excess excision of the underlying extra skin [23].

Every surgical procedure has advantages and disadvantages. Design can be tricky, there might be not enough correction, or scarring can occur in the medial canthal region. Therefore, it is best to choose an easy and less complicated surgical procedure dependent on surgeon experience [24].

\section{Burden factors}

Burden factors that do not cause double eyelids in Asians include: thick skin, subcutaneous fat tissue, OOM, orbital septum, orbital fat, lower positioned transverse ligament (LPTL), and tight lateral horn.

Typical Asian upper eyelids are thicker and have no double eyelids. When the eyes are open, skin, muscles, and fat droop down and push on the eyelashes due to these burden factors. In
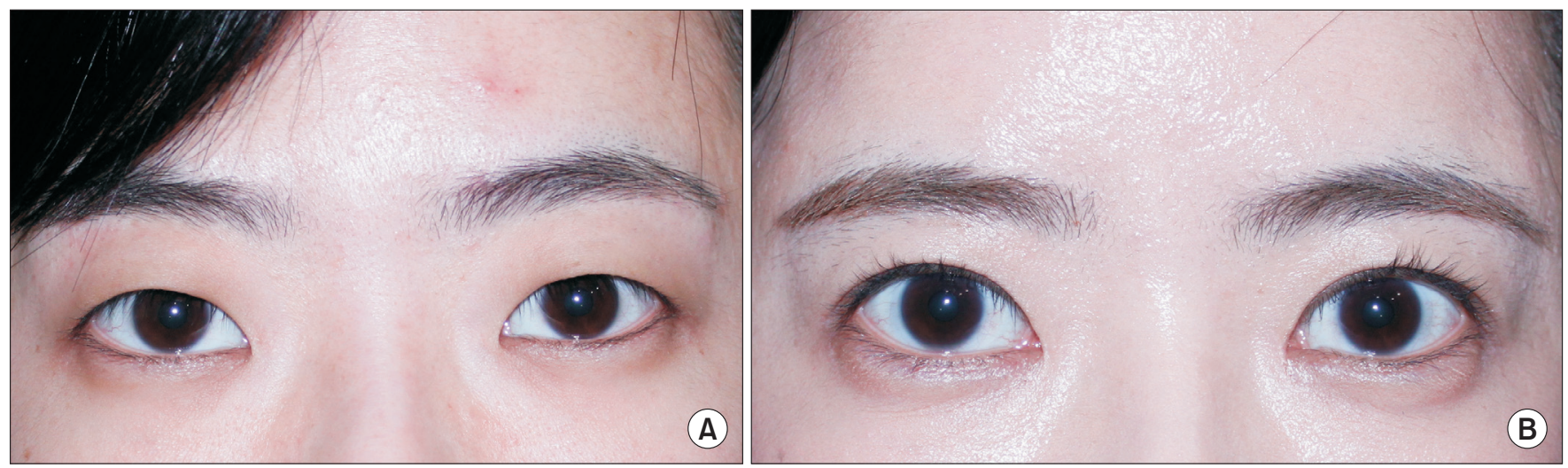

Fig. 9. (A) This 23-year-old woman has single eyelid with Park classification type III epicanthal fold. (B) This photo is taken 3 months after two partial incision technique combined with root-z medial epicanthoplasty. 

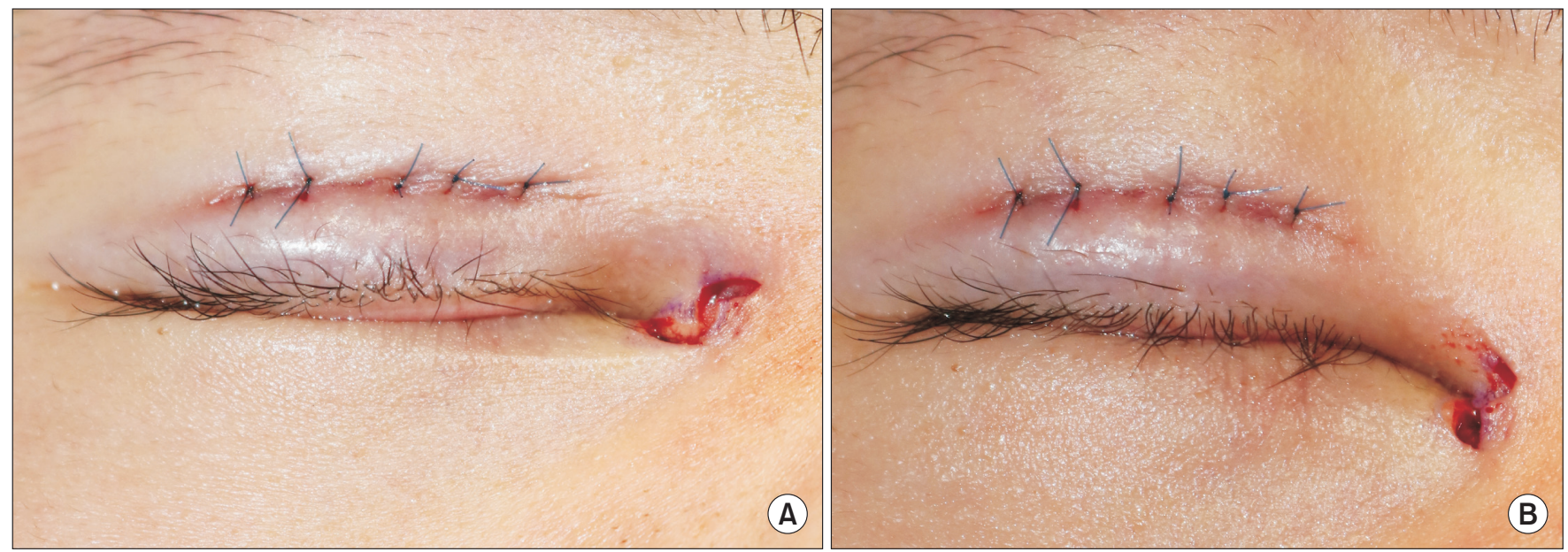

Fig. 10. (A) Z incision was made on epicanthal fold. (B) After releasing the tension on medial canthus, each flap naturally rotates.
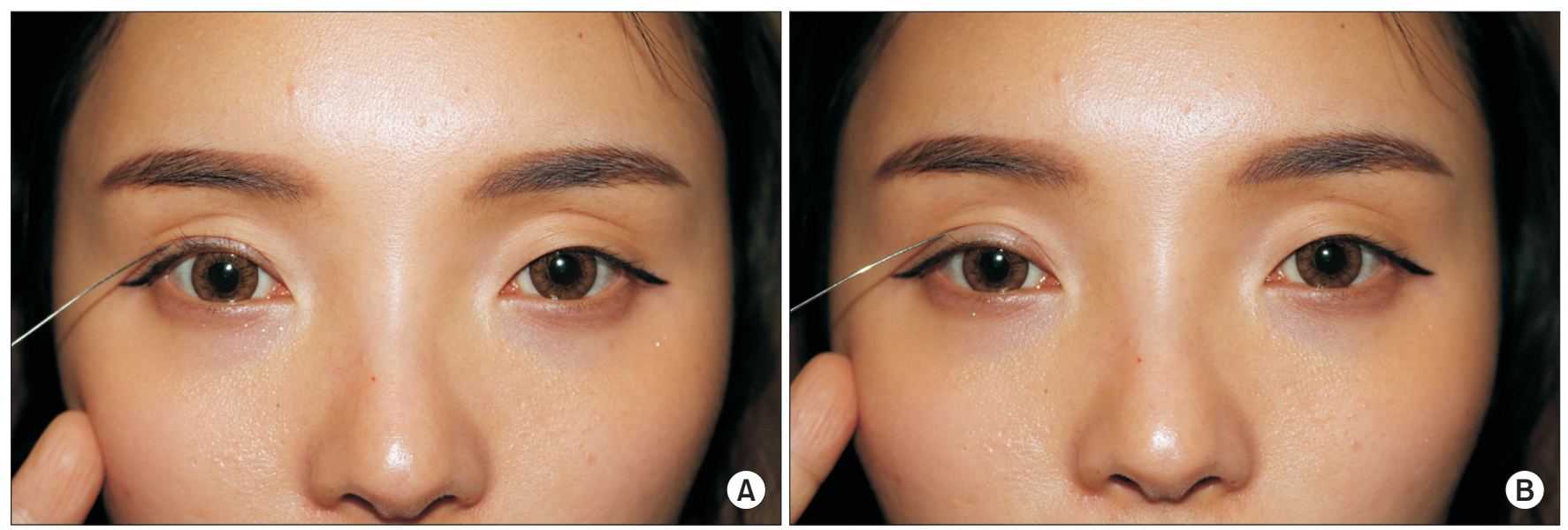

Fig. 11. (A) This photo shows in-fold type lid crease, which lies below the epicanthal fold. (B) This one shows out-fold type crease, which lies above the epicanthal fold.

upward gaze, only the posterior lamella, such as the tarsal plate, is pulled up, the anterior lamella such as skin and muscle sag further, and the eyelashes are pressed further. To avoid this discomfort, the frontalis muscle is used excessively when looking up and even during normal view. In order to create long-lasting, distinct, and beautiful double eyelids, it is of the utmost importance to properly eliminate these burden factors.

\section{Type of desired lid crease}

When the double eyelid touches the epicanthal fold, it is called on-fold crease. It is called out-fold crease when the double eyelid line goes up the epicanthal fold, and in-fold crease when the double eyelid line goes down the epicanthal fold [25] (Fig. 11).

Although the lid crease desired by most Koreans is in-fold or on-fold type, some patients request the out-fold type. However, the out-fold type is unnatural to Asians, and high supratarsal fixation with high lid crease can cause a sleepy appearance after surgery. Suhk et al.[25] reported that lid crease should be made out-fold type only if the palpebral fissure is more than 30 $\mathrm{mm}$, exophthalmos is present and there is no epicanthal fold, good levator function, minimal amount of orbital fat, no pretarsal fullness, and thin skin. To create an out-fold crease, the lid crease height of the medial side should be similar to the central side, and the incision line should be made longer medially.

\section{Choice of surgical technique and design of a lid crease}

The buried suture technique is a simple procedure that is well suited for the theory that adhesion between the dermis and the levator aponeurosis or tarsus is an important mechanism of lid 
crease formation. Recently, a variety of continuous suture techniques have been introduced that extend the range of adhesion between the two tissues through an alternate running suture. It is known that the incidence rate of suture loosening is lower than that of the interrupted suture technique [26].

It has been reported that the OOM and skin are thin in the area where lid crease are present [10]. Therefore, the OOM is appropriately removed by incision or resection to make it thinner and to induce more adhesion, resulting in a stronger double eyelid than with the buried suture technique. In addition, the buried suture technique alone might not properly correct for severe lash ptosis, and failure to properly remove the fat, the most important burden factor in the upper eyelid, can cause lid crease loosening over time after blepharoplasty. Unlike the buried suture technique in proper patients with thin skin and less fat, the double eyelid can fade or loosen unless it is properly corrected in patients with prominent burden factors such as thick skin, muscle, and fat. Therefore, if the burden factors are properly eliminated and the adhesion between the skin and the tarsus or levator aponeurosis is sufficient, the double eyelids can be prevented from fading or disappearing. However, regardless of the technique used to create double eyelids, they cannot be maintained $100 \%$. When allergies are present or contact lenses are constantly worn, eyelid edema can recur, and surgically created double eyelids can fade or disappear.

To create a natural dynamic lid crease, do not remove too much of the orbicularis muscle. Also, it is good to use the levator aponeurosis covering the tarsus, which is located under pretarsal OOM. Usually two to three buried sutures followed by skin-levator aponeurosis-skin sutures can form natural double eyelids.

In general, adhesion between the levator aponeurosis or tarsus and dermis is essential for the formation and maintenance of lid crease in Asians. However, the dermis of the eyelid is thin and is not easy to suture. When the suture is too superficially fixed, it can cause a cheese-wiring effect, and the adhesion between the tarsus and aponeurosis is likely to be lost. According to Choi and Eo et. al, adhesion of the levator aponeurosis and subcutaneous layer is essential for cosmetically satisfactory lid crease formation, and the fixation point is the outer fascia of orbicularis oculi muscle (OFOOM) [27].

At our clinic, two or three buried sutures are inserted to correct the direction of the eyelashes and to prevent the lid crease from loosening. The author once again attaches the levator aponeurosis to strengthen the adhesion when the skin is sutured.

\section{Preoperative preparation}

A thorough history in all patients is essential. Patients with systemic diseases that can affect the operation, such as hypertension, diabetes, hemorrhagic disease, and chronic diseases such as thyroid disease, should be carefully checked. Smoking, drinking, drug use, and psychiatric history should also be investigated. All medicines and foods that can increase the risk of bleeding, such as aspirin, warfarin, multivitamins, omega 3, herbal medicine, ginseng, soybean pills, garlic, moxa, and vinegar, should also be checked [11]. After consultation with the patient's physician, patients should discontinue aspirin at least one week before surgery, and other blood thinners should be discontinued 5 days before surgery.

If a patient has made artificial double eyelids using glue, or if there is persistent inflammation in the skin such as atopy, it is advisable to plan the surgery after first relieving the inflammation of the skin using steroid ointment.

\section{Operation}

\section{Surgical instruments and equipment}

Basic instruments and tools needed for surgery include: tissue forceps, No. 15 Bard-Parker blade, Wescott spring scissors, small sharp scissors, straight hemostat, locking needle holder, radio frequency $(\mathrm{RF})$ cautery machine, and 6-0 and 7-0 suture material (nylon, Prolene, black silk) (Fig. 12).

\section{Surgical procedures}

\section{Design}

With the patient in a sitting position, the surgeon checks the height of the lid crease line made by the lid crease maker (Fig.

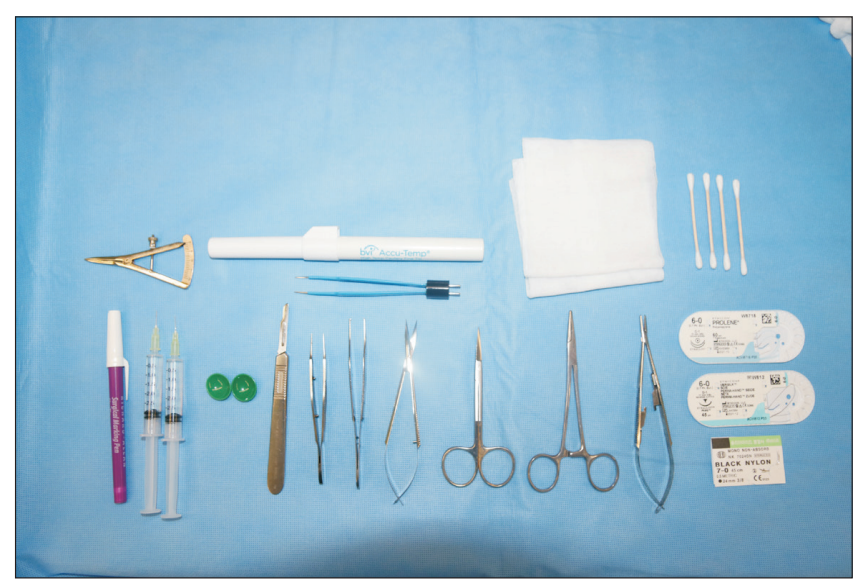

Fig. 12. Basic instruments and tools used for blepharoplasty. 


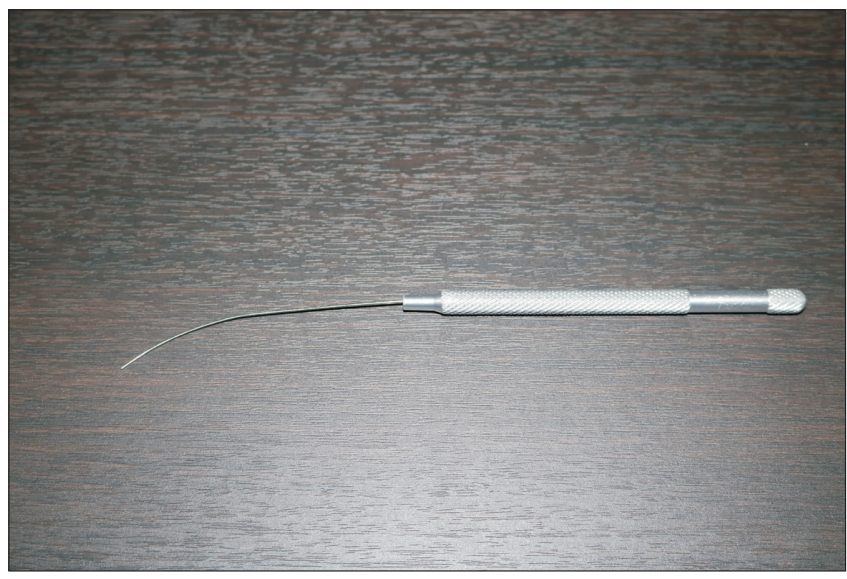

Fig. 13. Lid crease maker.

13) and draws the corresponding line with the marking pen where the desired lid crease is formed. The Korean preferred double eyelid shape is an in-fold type at over $90 \%$, and likes eyes that are not stuffy inside and not slanted. The size of the double eyelids is adjusted based on the height of the lid crease line. In other words, if you want a big double eyelid, you design a lid crease line at a higher position from the lid margin. However, in patients with severe dermatochalasis, if the lid crease line is too high, excessive high supratarsal fixation can result in a sleepylooking appearance. In such cases, it is better to lower the lid crease height and remove the skin.

\section{Local anesthesia}

For anesthesia, $2 \%$ lidocaine is mixed with 1: 100,000 diluted epinephrine, and the mixture is injected with a total dose of 1 to $1.5 \mathrm{cc}$ per eyelid. Using a 30 -gauge needle, the eyelid skin is pulled tightly to the lateral side, moving from the lateral side to the medial side, and the medicine is injected subcutaneously to minimize pain and bleeding. Generally, after waiting about 5 to 10 minutes after the local anesthesia injection, the operation is started. The surgeon can shorten the waiting time by squeezing the bulged eyelid with the thumb and index finger to spread the anesthetic around the operation site.

\section{Skin incision}

The upper eyelid skin was pulled outward near the lateral canthus to flatten the skin, and a one-step smooth incision was made with a No. 15 Bard-Parker blade. This can prevent the skin from being incised in a zigzag shape.

\section{OOM incision}

When the OOM is seen after the skin incision, the surgeon

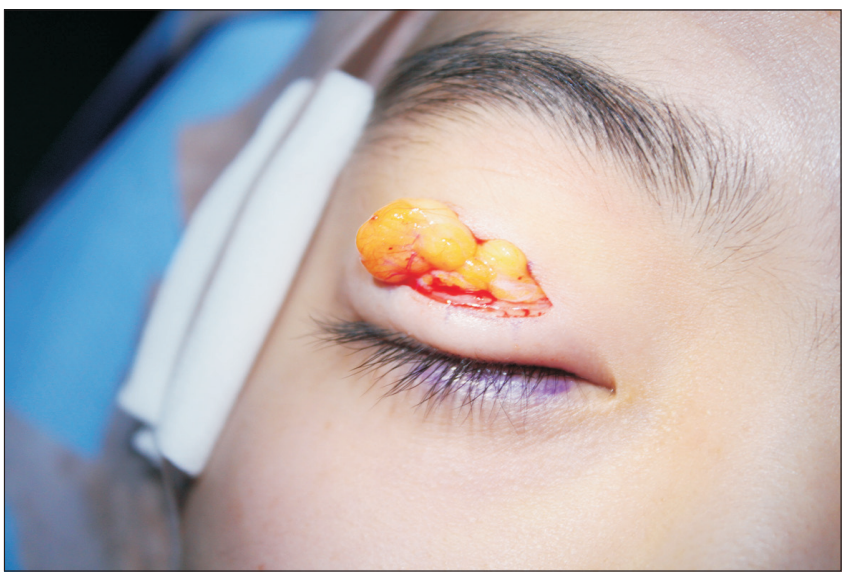

Fig. 14. Preaponeurotic fat is bulged out after cutting orbital septum.

pulls up the upper part of the OOM, and the assistant pulls up the lower part of the OOM with tissue forceps. Then it is dissected vertically using Westcott scissors.

\section{Orbital septum opening and orbital fat resection:}

When the surgeon opens the orbital septum, the bulged orbital fat is extruded (Fig. 14). Orbital fat, especially preaponeurotic fat, is a very important surgical landmark and is located over the levator aponeurosis. It is not easy to find orbital fat if the suborbicularis muscle fascia and septum are thick, fat is atrophied, or the fat is pulled upward. In this case, beginners can fail to identify levator aponeurosis, and occasionally damage the levator aponeurosis. After the orbital septum is opened, the yellow orbital fat that has been pushed out can be removed with Westcott scissors after being grabbed by a straight hemostat, and bipolar cautery is used to control the bleeding in the stump of orbital fat. Because the medial fat can be deeply entrapped after being cut, the hemostat should be loosened to check for bleeding while holding the stump with tissue forceps. The surgeon might find dense ligament tissue from the superomedial side to the inferolateral side above the levator aponeurosis, which is thought to be a thickened septum and is called a lower positioned transverse ligament (LPTL, Fig. 15). This structure is regarded as interfering with the opening of the eyes and, if found, will be cut vertically to reduce tension when the eye is open.

\section{Identification of the levator aponeurosis}

The next structure to identify is the levator aponeurosis. To confirm this structure, you can ask the patient to look up while holding the area thought to be the levator aponeurosis with tissue forceps. If you feel a pulling through the holding forceps and 


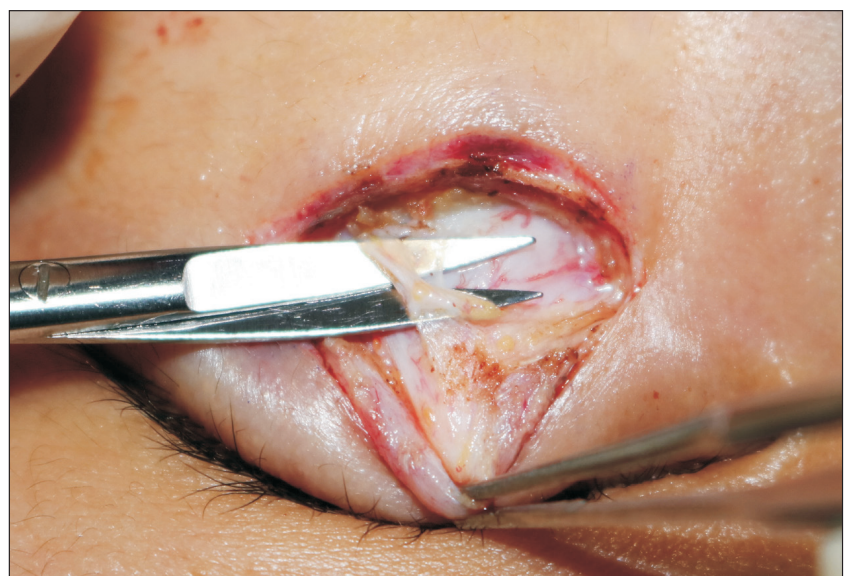

Fig. 15. This thick fibrous tissue is lower positioned transverse ligament (LPTL) which disturbs the eyelid opening.
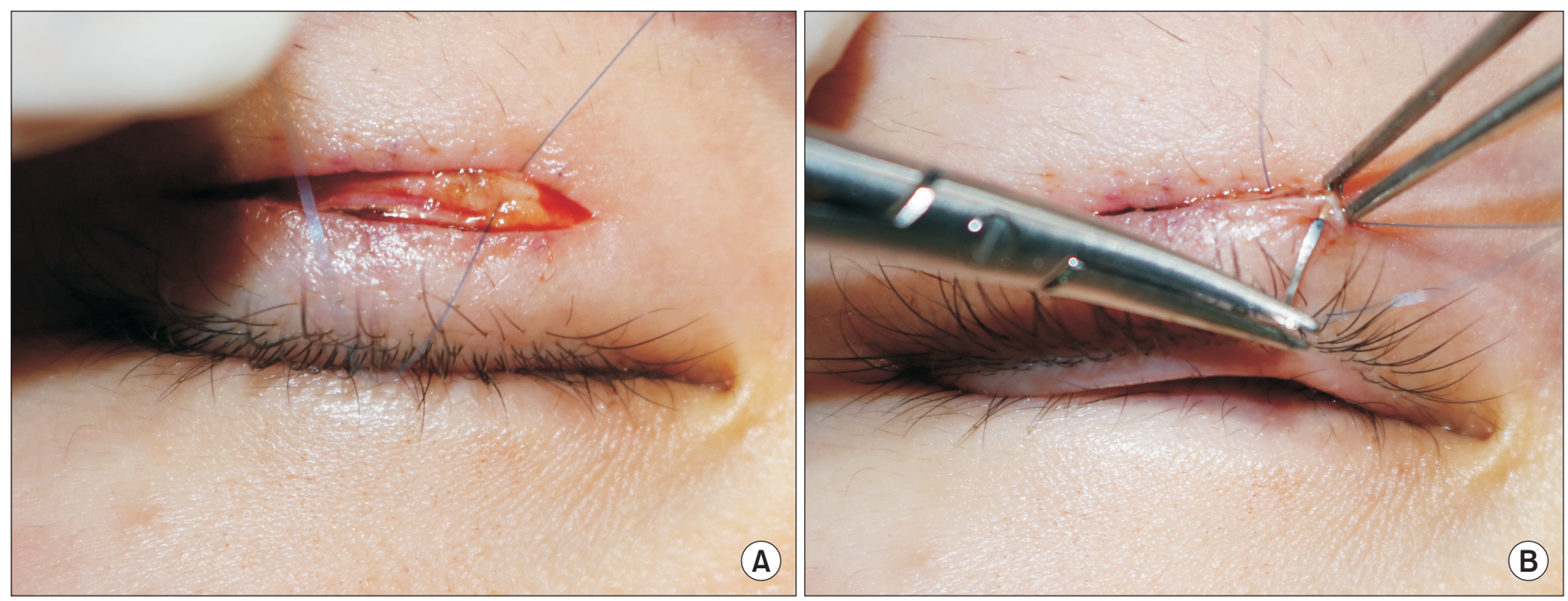

Fig. 17. (A) This photo shows levator aponeurosis suture with 6-0 blue nylon (B) and suturing the dermis of inferior flap to evert the eyelashes and make lid crease as well.

the patient cannot open his or her eyes, the tissue is the levator aponeurosis.

\section{Pretarsal OOM debulking}

The pretarsal OOM of the lower flap at the skin incision site should be appropriately removed to prevent sausage deformity (Fig. 16). Care should be taken because excessive resection can damage the levator aponeurosis that passes over the tarsal plate, which can cause postoperative ptosis.

\section{Lash everting suture technique}

Also called lid crease formation suturing, the lash everting suture technique is an important procedure during surgery. Its aim is to improve the functional and cosmetic outlook by

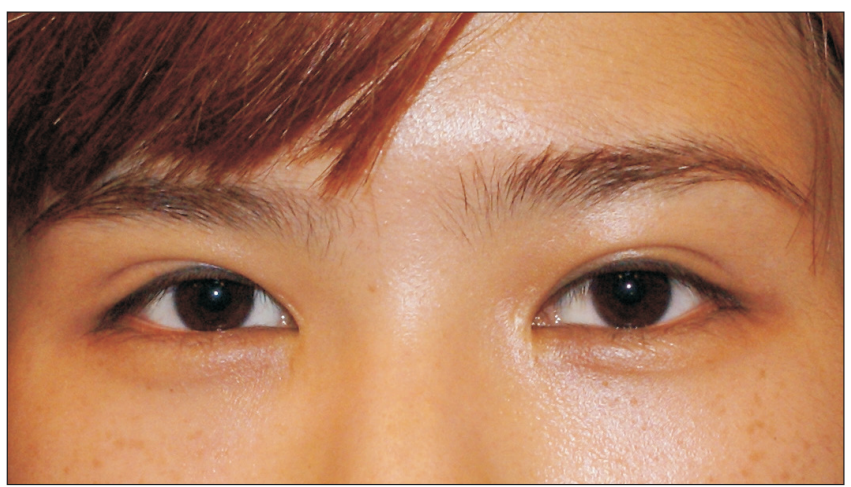

Fig. 16. This patient had upper blepharoplasty, but shows pretarsal fullness (called sausage deformity). producing an upward lash direction in order to maintain a clear lid crease line. Between the dermis of the lower flap of the skin incision site and the tarsus or the levator aponeurosis, 6-0 or 7-0 nylon sutures are fixed in two or three points (Fig. 17). Depending on the patient's condition, multiple sutures can be used to create a firm double eyelid. Suturing of the dermis of thin eyelid skin can be technically challenging, so it might be convenient to suture the OOM of the lower flap and the outer fascia of the OOM. When adjusting the fixation point and strength of the suture knot, it is advisable to slightly overcorrect to produce lash eversion when the patient is lying down. It is not good cosmetically if too much of the lid margins are exposed due to excess lash eversion. 


\section{Skin suturing}

Suture between skin-levator aponeurosis-skin using 6-0 or 7-0 nylon, Prolene, or black silk. During suturing, the direction of the eyelashes should be carefully checked. If the eyelashes are excessively everted and the lid margin is overexposed, direct skin-to-skin sutures should be done. 6-0 fast absorbing plain gut (FAPG) sutures do not need to be removed, so they will be useful in patients who are difficult to follow up or in uncooperative patients.

\section{Medial epicanthoplasty}

When the inner eyelid skin sags and the eyelashes are pressed tightly, the epicanthal fold should be resolved to obtain good cosmetic and functional results. If the epicanthal fold is not resolved, the eye can appear to be rounded, or the double eyelid line can be obscured to the inside, and the medial eyelashes might still be pressed downward. Therefore, if the epicanthal fold seems to have a cosmetic or functional effect on postoperative outcome, a medial epicanthoplasty should be performed. If the nasal dorsum is too low, medial epicanthoplasty alone might not be sufficient to achieve a good postoperative outcome. In that case, the nasal dorsum can be augmented by rhinoplasty or filler to achieve good cosmetic results.

\section{Postoperative care}

Two to three times a day, antibiotic ointment is applied on the skin suture site to prevent infection. To reduce swelling, bleeding, and pain, ice is applied around the operation site for as long as 3 days, including the day of surgery. However, direct irritation or rubbing on the operation side is not advised. Subsequently, warm massage can be used to ensure better blood supply to wound site.

Occasionally, when using nylon or Prolene, the thread can irritate the skin on the upper side, resulting in unwanted lid crease or poor eye opening. In this case, the thread can be shortened to prevent skin irritation by scissors. Black silk is soft and does not have this problem, but it causes more inflammation.

Asymmetry might be seen soon after surgery due to a difference in degree of swelling or recovery of the two eyes and will mostly be relieved with time.

When an unwanted double eyelid line develops at the early postoperative stage, it might be helpful to have a patient exercise up and down with his or her hands on the forehead.

If the patient continues to habitually use the forehead and finds that it is difficult to produce a clear double eyelid line, it can be helpful to immobilize the forehead by injecting Botox.

\section{Conclusion}

Thorough preoperative evaluation is essential for good results. The purpose of blepharoplasty for Asian eyelids is to improve functionality and cosmetic appearance by first, correcting the burden factors that do not cause double eyelids, and second, correcting the direction of eyelashes with a precise understanding of Asian eyelid anatomy. In Koreans, it is usually preferable to use an in-fold type of double eyelid and to perform medial epicanthoplasty when the medial curve of the upper eyelid is steep.

\section{Conflicts of interest}

The authors have nothing to disclose.

\section{References}

1. Mathews G, White B. Japan's changing generations: are young people creating a new society? London: RoutledgeCurzon; 2004. 206 p.

2. Kakizaki H, Malhotra R, Selva D. Upper eyelid anatomy: an update. Ann Plast Surg 2009;63:336-43.

3. Most SP, Mobley SR, Larrabee WF Jr. Anatomy of the eyelids. Facial Plast Surg Clin North Am 2005;13:487-92, v.

4. Park DH, Choi WS, Yoon SH, Song CH. Anthropometry of asian eyelids by age. Plast Reconstr Surg 2008;121:1405-13.

5. Sayoc BT. Absence of superior palpebral fold in slit eyes; an anatomic and physiologic explanation. Am J Ophthalmol 1956;42:298-300.

6. Bang YH, Chu HH, Park SH, Kim JH, Cho JW, Kim YS. The fallacy of the levator expansion theory. Plast Reconstr Surg 1999;103:1788-91; discussion 1792-3.

7. Liu D, Hsu WM. Oriental eyelids. Anatomic difference and surgical consideration. Ophthal Plast Reconstr Surg 1986;2:59-64.

8. Doxanas MT, Anderson RL. Oriental eyelids. An anatomic study. Arch Ophthalmol 1984;102:1232-5.

9. Jeong S, Lemke BN, Dortzbach RK, Park YG, Kang HK. The Asian upper eyelid: an anatomical study with comparison to the Caucasian eyelid. Arch Ophthalmol 1999;117:907-12.

10. Kakizaki H, Takahashi Y, Nakano T, Asamoto K, Ikeda H, Iwaki $\mathrm{M}$, et al. The causative factors or characteristics of the Asian double eyelid: an anatomic study. Ophthal Plast Recon- 
str Surg 2012;28:376-81.

11. Drolet BC, Sullivan PK. Evidence-based medicine: blepharoplasty. Plast Reconstr Surg 2014;133:1195-205.

12. Rhee SC, Lee SH. Attractive composite faces of different races. Aesthetic Plast Surg 2010;34:800-1.

13. Lee TE, Lee JM, Lee H, Park M, Kim KH, Baek S. Lash ptosis and associated factors in Asians. Ann Plast Surg 2010;65:40710.

14. Malik KJ, Lee MS, Park DJ, Harrison AR. Lash ptosis in congenital and acquired blepharoptosis. Arch Ophthalmol 2007;125:1613-5.

15. Yamamoto H, Morikawa K, Uchinuma E, Yamashina S. An anatomical study of the medial canthus using a three-dimensional model. Aesthetic Plast Surg 2001;25:189-93.

16. Yi SK, Paik HW, Lee PK, Oh DY, Rhie JW, Ahn ST. Simple epicanthoplasty with minimal scar. Aesthetic Plast Surg 2007;31:350-3.

17. Lew JM, Uhm KI, Kim IK, Hong SE. Modified double-eyelid operation method in epicanthal fold patient. J Korean Soc Plast Reconstr Surg 1995;22:1401-7.

18. Tark KC, Kim JM. Z-epicanthoplasty combined with double eyelidplasty: its indication, results \& pitfalls. J Korean Soc Plast Reconstr Surg 2002;29:515-20.

19. Duke-Elder S. System of ophthalmology. London: St. Louis,
MO: Mosby; 1958.

20. Jordan DR, Anderson RL. Epicanthal folds. A deep tissue approach. Arch Ophthalmol 1989;107:1532-5.

21. Kakizaki H, Ichinose A, Nakano T, Asamoto K, Ikeda H. Anatomy of the epicanthal fold. Plast Reconstr Surg 2012;130:494e5 e.

22. Kwon B, Nguyen AH. Reconsideration of the epicanthus: evolution of the eyelid and the devolutional concept of Asian blepharoplasty. Semin Plast Surg 2015;29:171-83.

23. Oh YW, Seul CH, Yoo WM. Medial epicanthoplasty using the skin redraping method. Plast Reconstr Surg 2007;119:703-10.

24. Hu X, Lin X, Ma G, Jin Y, Chen H, Chen X, et al. Two-Z-epicanthoplasty in a three-dimensional model of Asian eyelids. Aesthetic Plast Surg 2012;36:788-94.

25. Suhk JH, Kiranantawat K, Nguyen AH. Physical evaluation of the Asian blepharoplasty patient. Semin Plast Surg 2015;29:145-57.

26. Baek JS, Ahn JH, Jang SY, Chee E, Jang JW. Comparison between continuous buried suture and interrupted buried suture methods for double eyelid blepharoplasty. J Craniofac Surg 2015;26:2174-6.

27. Choi Y, Eo S. Outer fascia of orbicularis oculi muscle as an anchoring target tissue in double eyelid surgery. J Craniofac Surg 2016;27:322-7. 